\title{
IAA Producing Endophytic Fungus Fusarium oxysporum wlw Colonize Maize Roots and Promoted Maize Growth Under Hydroponic Condition
}

\author{
Asif Mehmood ${ }^{1}$, Muhammad Irshad ${ }^{1}$, Naeem Khan ${ }^{2}$, Muhammad Hamayun ${ }^{1}$, Ismail ${ }^{1}$, Husna ${ }^{1}$, Arooj \\ Javed $^{3}$ and Anwar Hussain ${ }^{1 *}$ \\ ${ }^{1}$ Department of Botany, Abdul Wali Khan University Mardan, Pakistan
}

${ }^{2}$ Department of Plant Sciences, Quaid-i-Azam University Islamabad, Pakistan

${ }^{3}$ Department of Botany, Hazara University Mansehra, Pakistan

*Corresponding author: Anwar Hussain, Department of Botany, Abdul Wali Khan University Mardan, Pakistan, E-mail: drhussain@awkum.edu.pk

Received date: June 20, 2018; Accepted date: July 02, 2018; Published date: July 16, 2018

Copyright: (C) 2018 Mehmood A, et al. This is an open-access article distributed under the terms of the Creative Commons Attribution License, which permits unrestricted use, distribution, and reproduction in any medium, provided the original author and source are credited.

Citation: Mehmood A, Khan N, Irshad M, Hamayun M, Ismail, et al. (2018) IAA Producing Endopytic Fungus Fusariun oxysporum wlw Colonize Maize Roots and Promoted Maize Growth Under Hydroponic Condition. Eur Exp Biol Vol. 8 No. 4:24.

\section{Abstract}

Indole-3-acetic acid (IAA) is known for their role in plant root interactions with microbial partners. Current study was focused on role of IAA as signal for colonization between endopytic fungus Fusariun oxysporum wlw and maize roots. Culture filtrate of the strain contained $31 \mu \mathrm{g} / \mathrm{mL}$ of IAA. Addition of tryptophan concentration ranging from 500 to $1000 \mu \mathrm{g} / \mathrm{mL}$ in culture medium significantly enhanced production of IAA by Fusariun oxysporum wlw. The strain effectively colonized the roots of maize and subsequently enhanced the growth and proliferation of host plant. In order to demined the role of IAA in root colonization by $F$. oxysporum we inhibited the biosynthesis of IAA by using IAA biosynthesis inhibitor which efficiently reduced te colonization of $F$. oxysporum in maize roots by $46 \%$ (foliar application) and $62 \%$ (root application) of the seedlings without yucasin (IAA biosynthesis inhibitor) treatment suggesting an IAA crosstalk between the two partners. Exogenous application of IAA restored the ability of endophyte $F$. oxysporum to colonize maize roots and significantly improved different growth parameters of maize seedlings. It is concluded that a molecular crosstalk of maize roots and endophytic $F$. oxysporum wlw is necessary for subsequent endophytic association between them.

Keywords: IAA; Fusariun oxysporum; Yucasin; Maize

\section{Introduction}

Colonization of endophytic fungi enhances the ecological adaptability of the plant by improving its tolerance against the biotic and abiotic stresses [1]. Endophytic fungi colonize the living plant tissue without causing damage, and include a very diverse group of fungi with different life history. Mutualistic endophytic fungi have been the focus of increasing interest worldwide, mainly because of their beneficial effects on plant suitability, such as tolerance to biotic and abiotic stresses, nutrient availability and increased growth [2]. Endophytic fungi provide a number of benefits to the host plants in various ways, such as promotion of growth, protection against diseases and pests, and augmenting absorption of minerals [3]. Fungal endophytes produce bioactive metabolites that promote the plant endophyte interaction [4]. Promoting plant growth is the most important effect of fungal symbiosis [5], however endophytic fungi promote plant growth by producing various secondary metabolites, including ammonia and plant hormones, particularly IAA $[3,6]$. Phytohormones are flexible signaling molecules affecting many physiological processes of plants development even when present at low concentrations [7]. The whole catalog of plant developmental process controls by hormones auxin and it is conceivable that some microbes also produce auxin [8]. Plants and microorganisms, including bacteria, algae and fungi, can produce IAA [9]. The role of microbial IAA in plant-microorganism interactions has received increasing attention recently. In addition, some studies show that IAA is a signaling molecule in microorganisms because it affects gene expression in several microorganisms [10]. Plants and their interactions with rhizosphere-related microbes such as fungi have always been an area of concern, as the knowledge of these processes may lead to environmentally-friendly farming practices. Roots produce a variety of organic compounds, including sugars, organic acids and vitamins [11]. These are then used as nutrients or signals for the fungal population. In contrast, fungi release iron carriers, volatile compounds, and plant hormones that may enhance plant growth, either directly or indirectly, by increasing the nutrient availability of their host [12]. IAA produced by fungi can induce lateral root formation and root hair development [13]. In addition, IAA produced by fungi can defeat pathogenic strains and disease progression by enhancing the plant's immune response [14]. Under in vitro conditions, it has recently been recognized that various endophytic fungi produce GAs and indole-3-acetic acid (IAA). The biosynthesis of indole-3-acetic acid (IAA) has not been well developed in fungi like bacteria [15]. However, it has recently been established that certain fungi, including endophytes, 
harbor them in their growth medium [16]. Indole-3-acetic acid (IAA) is considered to be a biologically active form of auxin its degree has been strongly regulated in plants through the interaction of several alleyways, involving the biosynthesis, oxidation and hydrolysis of IAA, as well as the binding of IAA to macromolecules such as carbohydrates and amino acids [17]. Auxin is often suggested to play a role in the cross-talk between plant and fungal signaling during ectomycorrhizal establishment [18]. IAA is a plant hormone known to play an important role in cell elongation, division and cell enlargement [19]. We have published an article regarding in vitro growth promotion by endndopytic Fusarium oxysporum wlw. Keeping the above discussion in view the current study was aim to identify the role of IAA in maize root colonization by endophytic Fusarium oxysporum wlw and host growth promotion under hydroponic condition.

\section{Materials and Methods}

The endopyte wlw was isolated from leaves of Withania sominfera and was identified as Fusarium oxysporum wlw [20]. The experiment was conducted in plant microbe interaction laboratory Abdul Wali Khan University Mardan, Pakistan. Maize variety GAUHER was obtained from national agriculture research center (NARC) Islamabad. The seedlings were allowed to grow for 14 days inside growth chamber (DAIHN Lab Tech) under controlled conditions (photoperiod 16/8 h and temperature $25^{\circ} \mathrm{C}$ at midday and $15^{\circ} \mathrm{C}$ at night; $390 \mathrm{ppm} \mathrm{CO} \mathrm{CO}_{2} ; 40 \%$ humidity). The $F$. oxysporum was applied to seedlings after two days in Hoagland's solution in the form of $1 \mathrm{~mL}$ of fungal spores suspension prepared by the hemacytometer and adjusted to an inoculum density of $106 \mathrm{~mL}^{-1}$ spores.

\section{Determination of Indole-3-acetic acid in culture filtrate}

The isolated strain was screened for IAA production by subjecting the $\mathrm{CF}$ to calorimetric assay using Salkowski reagent (150 mL concentrated $\mathrm{H}_{2} \mathrm{SO}_{4}, 250 \mathrm{~mL}$ distilled water, $7.5 \mathrm{~mL} 0.5$ $\mathrm{M} \mathrm{FeCl}_{3} \cdot 6 \mathrm{H}_{2} \mathrm{O}$ ) [21]. The strain was cultured in czapek broth media containing different concentrations of tryptophan (100, $500,1000 \mu \mathrm{g} / \mathrm{mL}$ ) at $28^{\circ} \mathrm{C}$ and $120 \mathrm{rpm}$ for seven days. For comparison, endophyte were also cultured in czapek broth having no tryptophan. After seven days, the culture were harvested and filtered through Whatman No 1 filter paper. The culture filterate was centrifuged at $11963 \mathrm{~g}$ for $2 \mathrm{~min}$ and to 1 $\mathrm{mL}$ of CF $2 \mathrm{~mL}$ of salkwoski reagent was added. The salkowski reagent was allowed to react with the IAA (in CF) at room temperature for $30 \mathrm{~min}$ in the dark. After incubation period, intensity of the pink color was determined by taking optical density of the mixture against the blank ( $2 \mathrm{~mL}$ blank media and 2 $\mathrm{mL}$ salkowski reagent) at $540 \mathrm{~nm}$ using a uv-vis spectrophotometer (PerkinElmer Lambda 25). Straight line equation was derived from the standard curve produced by plotting standard IAA (Sigma Aldrich) concentrations $(10,25,50$, $100,150,200,250,300 \mu \mathrm{g} / \mathrm{mL}$ ) against OD. The derived equation $\mathrm{Y}=0.0081 \mathrm{X}-0.0151$ was used to quantify IAA in the CF of endophyte.

\section{Effect of yucasin on release of IAA in culture filtrate and biomass production}

In order to examine the effect of yucasin (-5-(4chlorophenyl)-1,2-dihydro-1,2,4-triazole-3-thione) auxin biosynthesis inhibitor, the isolated endophyte was grown in czapek medium containing $(50 \mu \mathrm{M})$ of yucasin under conditions mentioned above. One week later, the cultures were collected and sieved with Whatman No 1 filter paper. The production of IAA in the filtrate was screened as previously described, and the biomass was collected and stored in a $50 \mathrm{~mL}$ Falcon tube at $60^{\circ} \mathrm{C}$ in an oven for 5 days. After five days, dry biomass was determined in the fractions.

\section{Maize growth in hydroponic system}

For the root colonization assay, $40 \mathrm{~mL}$ of half-strength Hoagland's solution [22,23], was taken in a $100 \mathrm{~mL}$ beaker. The truncated micropipette tip was held in sterile cardboard and sealed with a pumpkin faucet as Hydroponics set up [24]. Maize seed was surface sterilized using $\mathrm{HgCl}_{2}$ solution (0.1\%) and washed three times with distilled water. After surface sterilization, 5 seeds were placed in an autoclave petri plates with $2 x$ filter paper and incubated at $28^{\circ} \mathrm{C}$ for 5 days and allowed to germinate. The uniform seedlings were transferred to a hydroponic setting and the seedlings were inoculated with endophyte spores suspension in water adjusted to an inoculum density of $106 \mathrm{~mL}^{-1}$ spores. After two weeks of growth, seedlings were harvested and various growth parameters i.e shoot and root length, and dry weights were measured.

\section{Root colonization assay}

To determine fungal colonization in maize root plants were collected after 14 days, washed with tap water, cut into small pieces and stained with lectophenol cotton blue dye (Cotton blue $0.05 \mathrm{~g}$, phenol crystals $20 \mathrm{~g}$, glycerol $40 \mathrm{~mL}$, lactic acid 20 $\mathrm{mL}$, distilled water $20 \mathrm{~mL}$ ) for 20 mins. After $20 \mathrm{~min}$, the segments were washed and observe under light microscope. The level of colonization was quantified by plotting the root segments on PDA. Shortly, the root were surface sterilized with $0.1 \% \mathrm{HgCl}_{2}$ and washed with distill water and cut into $1 \mathrm{~cm}$ fragments. Six root segments for each plant, 2 from upper part (near to inoculum) two from middle part and two from lower part (near to stem) were kept on clean filter paper and inoculated on PDA plates and incubated for one week. After incubation for a week, colonization percentage was determined by dividing number of root segments positive for colonization by total number of root segments studied and multiplied by 100 . For comparison and validation of surface sterilization procedure, non-injured root segments were plated on PDA. Also, the root exudates of inoculated and non-inoculated seedlings were collected and screened for the determination of IAA, according to standard procedure as discussed previously.

\section{Exogenous application of IAA}

For the exogenous application of IAA, plants were grown in 40 $\mathrm{mL}$ half strength of Hoagland's solution in $100 \mathrm{~mL}$ beaker. After two days of growth in liquid solution, standard IAA at a final 
concentration of $10 \mu \mathrm{g} / \mathrm{mL}$ was applied through ariel spray on plants [25]. The seedlings were then inoculated with fungi as described earlier and uninoculated seedlings were used for comparison. After 14 days of growth the seedlings were harvested and various growth constraints via shoot length, root length, and dry weights were measured and roots exudates were screened for IAA.

\section{Inhibition of IAA biosynthesis by yucasin}

Biosynthesis and exudation of IAA by the root was inhibited by applying yucasin (50 $\mathrm{MM})$ [26], in the form of foliar spray. Root colonization by the endophyte was assayed in the presence of yucasin to assess the role of IAA in root-endophyte association.

\section{Statistical analysis}

Data was analyzed using SPSS for windows 16.0 (SPSS Inc., Chicago, IL, USA). An analysis of variance was performed to determine the effect of the treatment and the errors associated with the experiment. To identify significant differences between treatments, average comparisons were performed using the Duncan multiple-range test $(P=0.05)$, where the error of the mean squared error was used to estimate the standard error of the difference between the means.

\section{Results}

\section{Determination of IAA in culture filtrate of $F$. oxysporum}

The strain F. oxysporum wlw was able to secrete significant quantity $(31.6 \mu \mathrm{g} / \mathrm{mL})$ IAA in their culture medium. The culture of endophyte wlw exposed to yucasin (IAA biosynthesis inhibitor) had significantly lower amount of IAA than their culture grown in the absence of this inhibitor. The amount of IAA released by endophyte was reduced by up to $37 \%$ in yucasin containing media than the control culture. Addition of different concentrations of tryptophan (500 and $1000 \mu \mathrm{g} / \mathrm{mL}$ ) in the media affect the amount of IAA released by the endophyte wlw. Increasing tryptophan concentration from 500 to 1000 significantly enhanced the amount of IAA released in the culture by $17 \%$ and $25 \%$ respectively (Figure 1 ).

\section{Endophyte growth response to exogenously applied yucasin}

Application of yucasin was inhibitory to endophyte growth as indicated by lower dry biomass (Figure 2). Yucasin exposed culture had significantly lowered (30\%) the biomass than in culture having no inhibitor.

\section{Effect yucasin, IAA and endophyte $F_{0}$ oxysporum on maize growth}

Exogenous application of yucasin in the form of foliar spray or in Hogland's media (Y-R) retarded shoot length by $28 \%$ and $12 \%$ in comparison to control. As was expected, exogenous application of phytohormone IAA significantly enhanced shoot length by $40 \%$ as compared to control. Also the shoot growth of maize seedlings was enhanced significantly in media containing endophyte wlw. Endopyte wlw improved shoot length by $26 \%$ as compared to the control. As noted above, shoot growth declined in seedlings exposed to exogenously applied yucasin. However, endophyte wlw associated seedlings were resistant to this inhibitor and showed normal growth even in the presence of yucasin. As renowned above, seedlings growth was promoted by exogenous application of IAA. However growth of maize seedlings was enhanced further in media containing endophyte in binary combination with IAA. Exogenously applied IAA synergistically interacted with endophyte wlw in promoting shoot length as evident from up to $13 \%$ greater shoot length in media containing a binary combination of IAA and endophyte than the media containing IAA without endophyte (Figure 3a). Root growth of maize seedlings was also negatively influenced by exogenously applied yucasin either as foliar spray or root entries. For instance, exogenous application of yucasin in the form of foliar spray caused $30 \%$ reduction in root length whereas root treatment of yucasin reduced root length by $31 \%$ in comparison to control. Exogenous application of IAA significantly improved root length by $22 \%$ as compared to control.

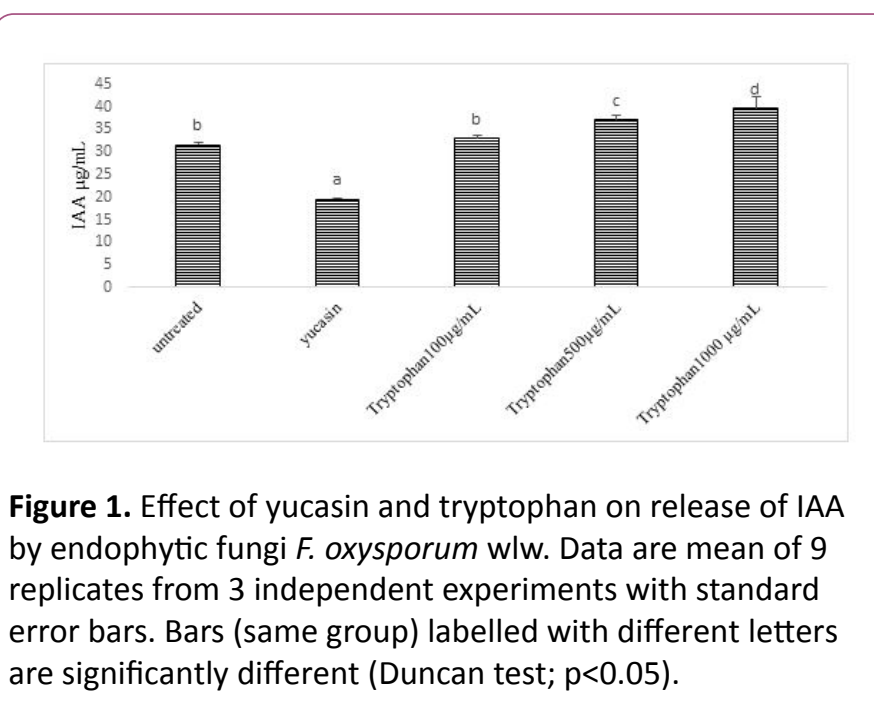

Root length was also significantly enhanced by endophyte wlw, where an increase of $60 \%$ over control was noticed. As noted above, exogenous application of yucasin declined seedlings root growth. However, endophyte associated seedlings were resistant to this inhibitor and showed normal growth even in the presence of yucasin. Similarly endophyte associated seedlings treated with foliar IAA had more roots than seedlings treated with foliar IAA alone (Figure $3 b$ ). Application of both foliar and root entries of yucasin reduced (69\%) seedlings dry biomass in comparison to control. Likewise exogenously applied IAA interacted with endophyte wlw and enhanced dry biomass significantly. For instance, seedlings that received binary combination of endophyte wlw and foliar IAA had 56\%, greater biomass than seedling received foliar IAA alone. Dry biomass was also enhanced significantly by the endophytes as compared to the control. Likewise seedling hosting endopyte $F$. oxyporum 
in binary combination with foliar yucasin had $27 \%$ greater biomass than foliar yucasin alone (Figure $3 \mathrm{c}$ ).

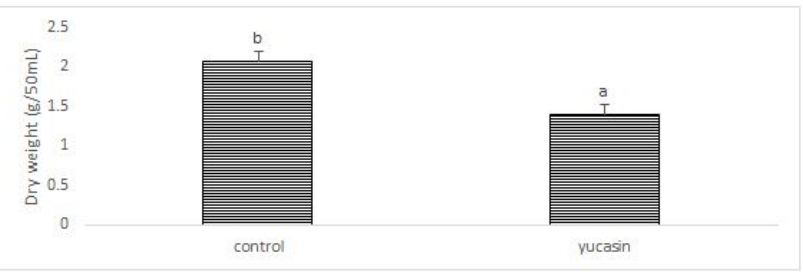

Figure 2. Effect of yucasin on the biomass of endophytic fungi F. oxysporum wlw. Data are mean of 9 replicates from 3 independent experiments with standard error bars. Bars (same group) labelled with different letters are significantly different (Duncan test; $p<0.05$ ).

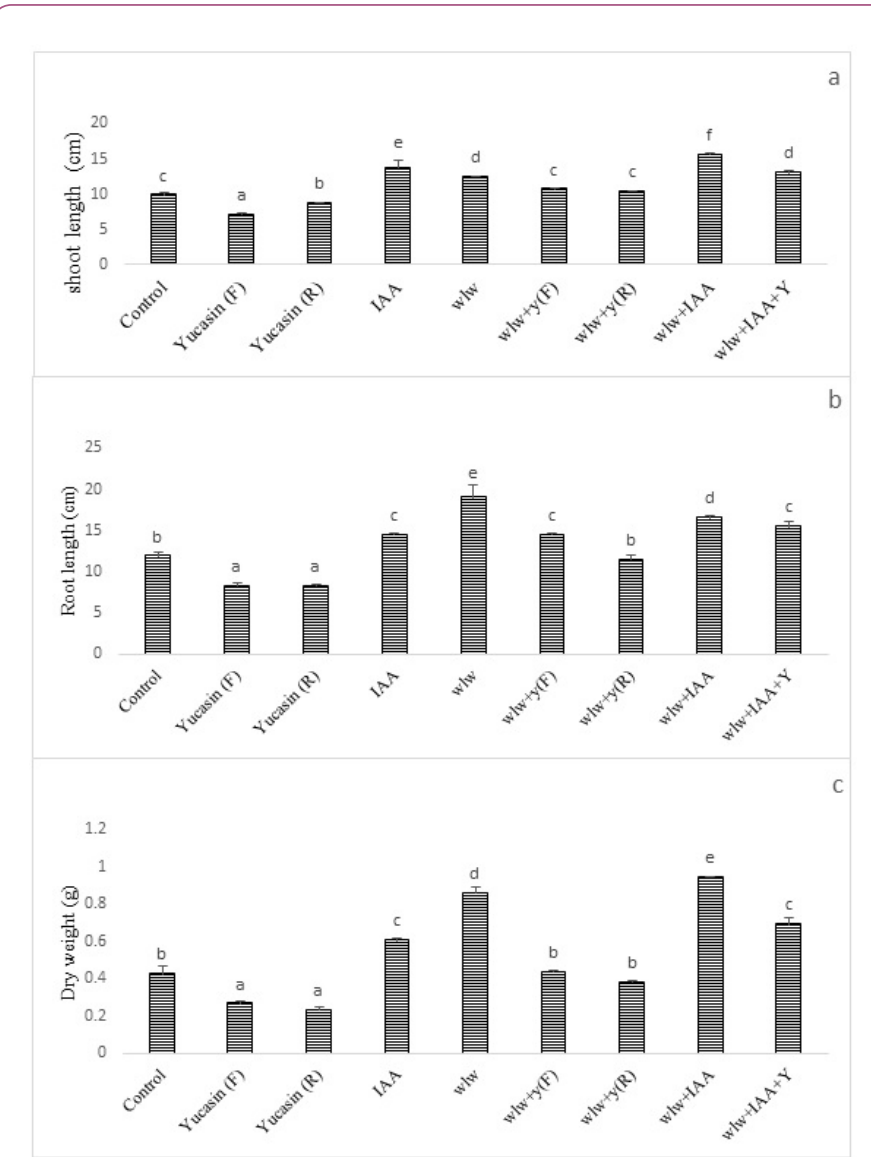

Figure 3. Effect of endophyte association (F. oxysporum), IAA (foliar), yucasin (foliar, $F$ and root applied, R) on the (a) shoot length, (b) root length and (c) dry biomass of maize seedlings grown hydroponically in Hogland's solution for 2 weeks. Data are mean of 9 replicates from 3 independent experiments with standard error bars. Bars labelled with different letters are significantly different (Duncan test; $p<0.05$ ).

\section{Effect of exogenously applied IAA and yucasin on exudation of IAA by $F_{0}$ oxysporum associated maize roots}

Highest amount of IAA was found in root exudates collected from endophyte associated maize seedling (10.5 \pm 1.6 to $12.5 \pm$ 1.8), no matter whether they received exogenous IAA or not. On exposure to yucasin (foliar or root), the seedlings ability to exude IAA was drastically reduced in comparison to the control. Interestingly, both foliar and root application of yucasin was inhibitory to the release of IAA by maize roots. In yucasin treated seedling, endophyte $F$. oxysporum restore the amount of IAA to the level of control seedlings (Table 1 ).

Table 1. Effect of IAA and yucasin (foliar, $F$ and root applied, R) on the exudation of a) IAA by maize roots associated with endophyte $F$. oxysporum. Seedlings inoculated with isolated strain and in control were left for growth for 2 weeks. Absorbance was compared with standard curve to find out concentration of IAA and sugars in samples. Data are mean of 9 replicates \pm standard error of mean. Similar bars labelled with different letters are significantly different (Duncan multiple range test; $p<0.05)$.

\begin{tabular}{|l|l|}
\hline Treatments & IAA $(\boldsymbol{\mu g} / \mathrm{mL})$ \\
\hline Control & $6.9 \pm 0.8 \mathrm{c}$ \\
\hline Yucasin (Foliar) & $1.8 \pm 0.0 \mathrm{a}$ \\
\hline Yucasin (Root) & $2.0 \pm 0.0 \mathrm{a}$ \\
\hline IAA & $10.1 \pm 1.6 \mathrm{~d}$ \\
\hline wIw & $10.5 \pm 1.6 \mathrm{~d}$ \\
\hline wIw+Y (F) & $6.6 \pm 0.7 \mathrm{c}$ \\
\hline WIW+Y (R) & $6.4 \pm 0.4 \mathrm{c}$ \\
\hline WIW+IAA & $12.5 \pm 1.8 \mathrm{e}$ \\
\hline WIW+IAA+Y & $11.3 \pm 1.0 \mathrm{e}$ \\
\hline
\end{tabular}

\section{Effect of IAA, yucasin on maize root colonization by endophyte wlw}

Colonization frequency of wlw in maize root varied among different root zones (Figures 4 and 5). Root zone of division attracted the endophyte with highest efficiency showing a colonization frequency of $66.6 \%$. However, colonization frequency dropped in the root above this zone and zone of elongation had a colonization frequency of $60.2 \%$. Area of root in the proximity of stem i.e. zone of maturation was the least colonized showing a colonization frequency of $25 \%$ only. Application of IAA in combination with the endophyte had even more pronounced effect on root colonization by the endophyte enhancing colonization frequency to $75 \%$ in the zone of cell division, $57.6 \%$ in the zone of elongation and $25.5 \%$ in the zone of maturation. Application of yucassin (IAA inhibitor) negatively influenced the root colonization of $w 1 w$ strain reducing its colonization frequency to $46 \%$ (foliar application) and $62 \%$ (root application) of the seedlings receiving no yucasin. 


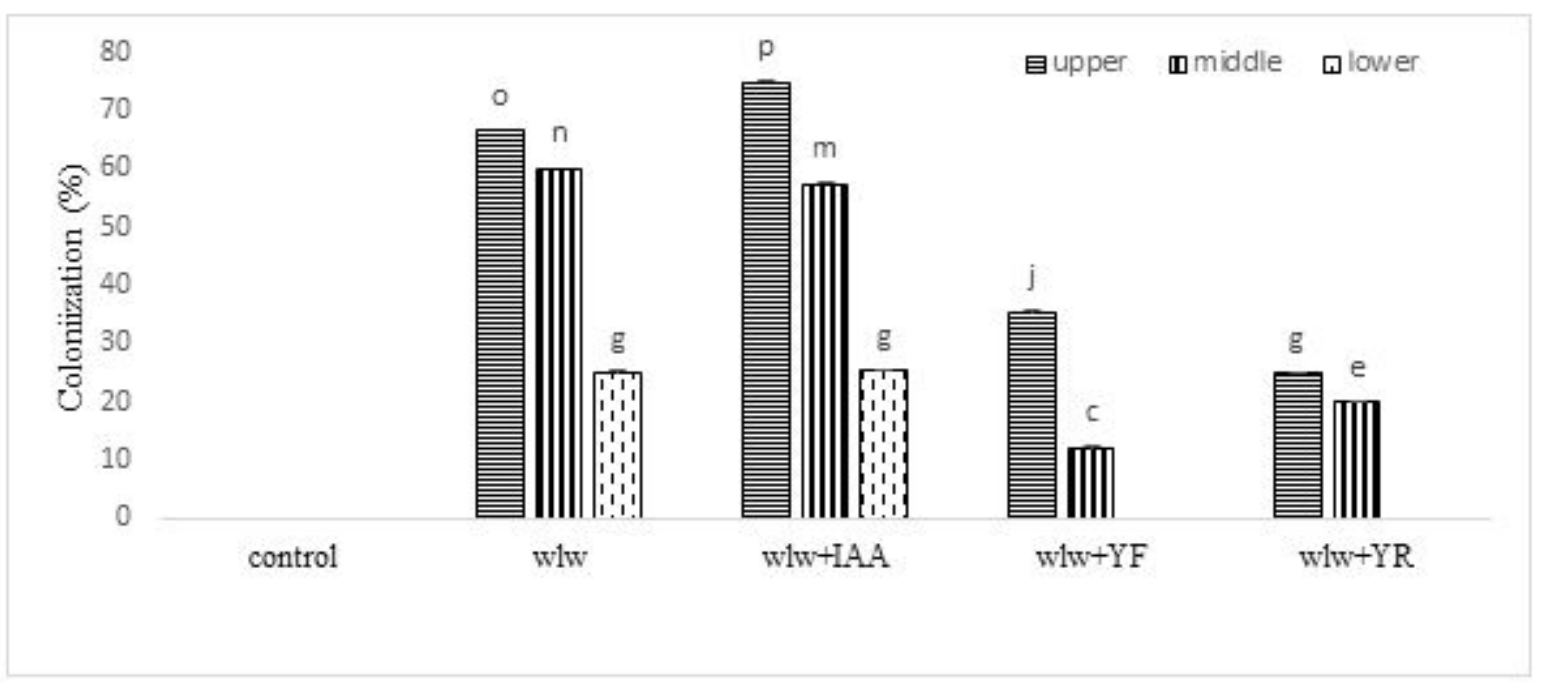

Figure 4. Effect of (a) yucasin (Y) and IAA on the mean percent colonization of maize roots by endophyte $F$. oxysporum wlw. The inhibitor were applied in the form of foliar spray (F) or in the culture media (R). Other treatments included exogenously applied IAA. The level of colonization was quantified by plotting the root segments on PDA after 14 days of co-culturing. Six root bricks for each plant, 2 from superior part (near to inoculum) two from middle part and two from inferior part (near to stem) were kept on clean filter paper and inoculated on PDA plates and incubated for one week. After a week colonization percentage was recorded according to the expression. Percent colonization of endophytic fungi=Number of positive segments/total number of root segments studied*100.

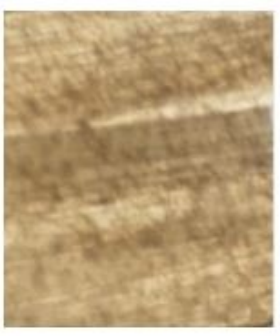

a

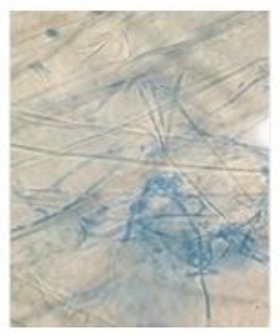

$\mathrm{b}$

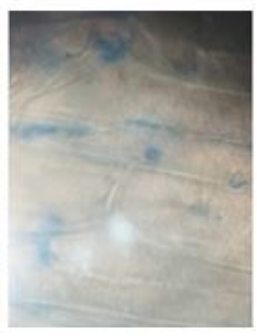

Figure 5. Visualization of wlw colonization (blue stained hyphae with cotton blue dye) in the maize root (a) control, (b) endophyte (c) endophyte+ yucasin.

\section{Discussion}

In present study an endophyte wlw was isolated from leaves of Withania somnifera previously and was identified as Fusarium oxysporum by comparative homology of ITS region near the $18 \mathrm{~S}$ rDNA gene [20]. The strain Fusarium oxysporum was also previously isolated as endophyte from Juniperus recurva [27]. The isolated fungus $F$. oxysporum possess plant growth promoting activities like ammonia and IAA production $[20,28]$. Fungal endophytes yield bioactive metabolites that promote the plant endophyte interaction [4]. Promotion of plant growth is the most important effect of fungal symbiosis [5]. Endophytic fungi promote plant growth by producing various secondary metabolites, including ammonia and plant hormones, particularly IAA $[29,30]$. Indole acetic acid as an essential compound for the growth and development of shoot and roots, many microorganism including plant growth promoting rizobacteria (PGPR) produce IAA [31]. Soil fungi and endophytes produce plant growth promoting substances IAA and gibberellin [7]. Culture filtrate of the endophyte $F$. oxysporum contained $(31.6 \mu \mathrm{g} / \mathrm{mL})$ of IAA and was greater than IAA recorded in culture filtrate of $P$. glomerata and Penicillium sp [16]. The endophyte $F$. oxysporum ability to produce IAA was tryptophan dependant demonstrating the presence of IPA (indole-3-pyruvic acid) pathway for the biosynthesis of IAA [32], described IPA, IAM (indole-3acetamide), and TRA (tryptamine), pathways in Fusarium delphinoides [31], reported IAM, IPA pathways in Colletotrichum gloeosporioides. Tryptophan is considered as a precursor for IAA biosynthesis and its addition of in culture medium enhances IAA production [33,34]. According to our result increasing tryptophan concentration from 500 and 1000 $\mu \mathrm{g} / \mathrm{mL}$ significantly enhanced amount of IAA by $17 \%$ and $25 \%$ greater than the control. Our results are in agreement with the work of Khalid et al. [35], who studied the effect of L-tryptophan concentration for the production of IAA and observed that Ltryptophan-derived auxin biosynthesis was enhanced several folds. Production of IAA has been observed in several endophytic fungi during the recent years $[7,16,36]$. In present study increasing tryptophan concentraion lead to significant incease in production of IAA by $F$. oxysporum. Indole-3-acetic acid (IAA) with or without precursor L-tryptophan, is produced by most genus of Trichoderma [37]. Production of IAA is thought to be a unique character enabling endophytes to promote plant growth [38]. In present study F. oxysporum was able to colonize maize roots and significantly enhanced shoot and root length and seedling dry biomass $(P>0.05)$. Increase in shoot and root 
length in fungal inoculated plants had been reported previously by many authors $[39,40]$. Also previously Mehmood et al. [20] reported increase in shoot and root length and seedling dry biomass by culture filtrate of endophytic Fusarium oxysporum. Previously Machungo et al., [41] reported that endophytic $F$. oxysporum increase plant height $11 \%$ which was lower than increase in shoot length caused by our isolate. Enhanced plant growth through endophytes may be the result of phytohormones produced by fungal endophytes [42]. Application of IAA in the form of aerial spray on corn seedlings enhanced the ability of roots to attract endophytes and their subsequent colonization in the roots. IAA contents increased in root exudates sprayed with IAA and inoculated with $F$. oxysporum. Previously, it was shown that exogenous application of IAA restored in vitro growth as well as efficiency of colonization in rice roots by mutant Nostoc spp. [43]. To verify the role of IAA in root-endophyte interaction, IAA biosynthesis was inhibited in plant by foliar application of yucasin [26]. Seedlings exposed to yucasin release significantly low amount of IAA confirming the inhibition of it biosynthesis. Interestingly, low IAA exuding maize roots were least colonized by the endophyte F. oxysporum pointing a role of this phytohormone in plantendophyte interaction.

\section{Conclusions}

The endophytic fungus F. oxysprum wlw promoted the growth of maize seedlings under under hydroponic condition. Its ability to generate IAA is responsible for colonizing the roots of corn and establishing beneficial symbiotic association. Inhibition of IAA limits their ability to interact and carry out symbiotic associations.

\section{References}

1. Schulz B, Boyle C (2005) The endophytic continuum. Mycological research 109: 661-686.

2. Combes A, Ndoye I, Bance C, Bruzaud J, Djediat C, et al. (2012) Chemical communication between the endophytic fungus Paraconiothyrium variabile and the phytopathogen Fusarium oxysporum. PLoS One 7: 47313.

3. Sieber TN, Waisel $Y$, Eshel A, Kafkafi U (2002) Fungal root endophytes. Plant roots: the hidden half 887-917.

4. Strobel GA (2003) Endophytes as sources of bioactive products. Microbes and infection 5: 535-544.

5. Hassan SE, Hijri M, St-arnaud M (2013) Effect of arbuscular mycorrhizal fungi on trace metal uptake by sunflower plants grown on cadmium contaminated soil. New biotechnology 30: 780-787.

6. Khan AL, Hamayun M, Kang SM, Kim YH, Jung HY, et al. (2012) Endophytic fungal association via gibberellins and indole acetic acid can improve plant growth under abiotic stress: an example of Paecilomyces formosus LHL10. BMC microbiology 12: 3.

7. Johansson O (2015) Plant pathogen defense: Signalling, resistance and cell death.

8. Spaepen S, Vanderleyden J, Remans R (2007) Indole-3-acetic acid in microbial and microorganism-plant signaling. FEMS microbiology reviews 31: 425-448.
9. Yuan ZC, Haudecoeur E, Faure D, Kerr KF, Nester EW (2008) Comparative transcriptome analysis of Agrobacterium tumefaciens in response to plant signal salicylic acid, indole-3acetic acid and $\gamma$-amino butyric acid reveals signalling cross-talk and Agrobacterium-plant co-evolution. Cellular microbiology 10: 2339-2354.

10. Fu SF, Wei JY, Chen HW, Liu YY, Lu HY, et al. (2015) Indole-3-acetic acid: A widespread physiological code in interactions of fungi with other organisms. Plant signaling \& behavior 10: e1048052.

11. Huang XF, Chaparro JM, Reardon KF, Zhang R, Shen Q, et al. (2014) Rhizosphere interactions: root exudates, microbes, and microbial communities. Botany 92: 267-275.

12. Contreras-cornejo HA, Macias-rodriguez L, Cortes-penagos C, Lopez-bucio J (2009) Trichoderma virens, a plant beneficial fungus, enhances biomass production and promotes lateral root growth through an auxin-dependent mechanism in Arabidopsis. Plant physiology 149: 1579-1592.

13. Ludwig-muller J (2015) Bacteria and fungi controlling plant growth by manipulating auxin: balance between development and defense. Journal of plant physiology 172: 4-12.

14. Kumla J, Suwannarach N, Bussaban B, Matsui K, Lumyong S (2014) Indole-3-acetic acid production, solubilization of insoluble metal minerals and metal tolerance of some sclerodermatoid fungi collected from northern Thailand. Annals of microbiology, 64: 707-720.

15. Waqas $M$, Khan AL, Kamran $M$, Hamayun $M$, Kang $S M$, et al. (2012) Endophytic fungi produce gibberellins and indoleacetic acid and promotes host-plant growth during stress. Molecules 17: 10754-10773.

16. Pieterse CM, Leon-reyes A, Van der ent S, Van wees SC (2009) Networking by small-molecule hormones in plant immunity. Nature chemical biology 5: 308.

17. Felten J, Martin F, Legue V (2012) Signalling in ectomycorrhizal symbiosis. Signaling and Communication in Plants 11.

18. Ishida Y, Nakamura A, Mitani Y, Suzuki M, Soeno K, et al. (2013) Comparison of indole derivatives as potential intermediates of auxin biosynthesis in Arabidopsis. Plant Biotechnology 30: 185-190.

19. Khan N, Bano A, Zandi P (2018) Effects of exogenously applied plant growth regulators in combination with PGPR on the physiology and root growth of chickpea (Cicer arietinum) and their role in drought tolerance. Journal of Plant Interactions, 13: 239-247.

20. Mehmood A, Irshad M, Husna, Ahmad A, Hussain A (2018) In Vitro Maize Growth Promotion by Endophytic Fusarium Oxysporum WLW. J Applied Environmental and Biological Sciences 8: 30-35.

21. Reversat G, Boyer J, Sannier C, Pando-bahuon A (1999) Use of a mixture of sand and water-absorbent synthetic polymer as substrate for the xenic culturing of plant-parasitic nematodes in the laboratory. Nematology 1: 209-212.

22. Barac T, Taghavi S, Borremans B, Provoost A, Oeyen L, et al. (2004) Engineered endophytic bacteria improve phytoremediation of water-soluble, volatile, organic pollutants. Nature biotechnology 22: 583.

23. Dardanelli MS, Manyani H, González-barroso S, Rodríguez-carvajaL MA, GIL-Serrano AM, et al. (2010) Effect of the presence of the plant growth promoting rhizobacterium (PGPR) Chryseobacterium balustinum Aur9 and salt stress in the pattern of flavonoids exuded by soybean roots. Plant and soil 328: 483-493. 
24. Vandeleur RK, Sullivan W, Athman A, Jordans C, Gllliham M, et al. (2014) Rapid shoot-to-root signalling regulates root hydraulic conductance via aquaporins. Plant, Cell \& Environment 37: 520-538.

25. Nishimura T, Hayashi KI, Suzuki H, Gyohda A, Takaoka C, et al (2014) Yucasin is a potent inhibitor of YUCCA, a key enzyme in auxin biosynthesis. The Plant Journal 77: 352-366.

26. Khan N, Bano A, Rahman M A, Rathinasabapathi B, Babar MA (2018) UPLC-HRMS-based untargeted metabolic profiling reveals changes in chickpea (Cicer arietinum) metabolome following longterm drought stress. Plant, cell \& environment 1-18.

27. Kour A, Shawl AS, Rehman S, Sultan P, Qazi PH, et al. (2008) Isolation and identification of an endophytic strain of Fusarium oxysporum producing podophyllotoxin from Juniperus recurva. World Journal of Microbiology and Biotechnology 24: 1115-1121.

28. Khan N, Bano A, Babar MA (2017) The root growth of wheat plants, the water conservation and fertility status of sandy soils influenced by plant growth promoting rhizobacteria. Symbiosis 72 : 195-205.

29. Fouda AH, Hassan SED, Eid AM, Ewais EED (2015) Biotechnological applications of fungal endophytes associated with medicinal plant Asclepias sinaica (Bioss.). Annals of Agricultural Sciences 60: 95-104.

30. Strobel G, Daisy B, Castillo U, Harper J (2004) Natural products from endophytic microorganisms. Journal of Natural products 67: 257-268.

31. Robinson M, Riov J, Sharon A (1998) Indole-3-Acetic Acid Biosynthesis in Colletotrichum gloeosporioides f. $\mathrm{sp}$. aeschynomene. Appl Environ Microbiol 64: 5030-5032.

32. Kulkarni GB, Sanjeevkumar S, Kirankumar B, Santoshkumar M, Karegoudar T (2013) Indole-3-acetic acid biosynthesis in Fusarium delphinoides strain GPK, a causal agent of wilt in chickpea. Appl Biochem Biotechnol 169: 1292-305.

33. Ahmad F, Ahmad I, Khan MS (2005) Indole acetic acid production by the indigenous isolates of Azotobacter and fluorescent
Pseudomonas in the presence and absence of tryptophan. Turkish Journal of Biology 29: 29-34.

34. Hoffman MT, Gunatilaka MK, Wijeratne K, Gunatilaka L, Arnold AE (2013) Endohyphal bacterium enhances production of indole-3acetic acid by a foliar fungal endophyte. PLoS One 8: e73132.

35. Khalid A, Arshad M, Zahir ZA (2004) Screening plant growthpromoting rhizobacteria for improving growth and yield of wheat. J Appl Microbiol 96: 473-480.

36. Sun PF, Fang WT, Shin LY, Wei JY, Fu SF, et al. (2014) Indole-3-acetic acid-producing yeasts in the phyllosphere of the carnivorous plant Drosera indica L. PloS one 9: e114196.

37. Waqas M, Khan AL, Lee IJ (2014).Bioactive chemical constituents produced by endophytes and effects on rice plant growth. Journal of plant interactions, 9: 478-487.

38. Hamayun M, Khan SA, Iqbal I, Ahmad B, Lee IJ (2010) Isolation of a gibberellin-producing fungus (Penicillium sp. MH7) and growth promotion of Crown daisy (Chrysanthemum coronarium). Journal of microbiology and biotechnology 20: 202-207.

39. Meletiadis J, Meis JF, Mouton JW, Verweij PE (2001) Analysis of growth characteristics of filamentous fungi in different nutrient media. Journal of Clinical Microbiology, 39: 478-484.

40. Schubert M, Dengler V, Mourad S, Schwarze FWMR (2009) Determination of optimal growth parameters for the bioincising fungus Physisporinus vitreus by means of response surface methodology. Journal of applied microbiology 106: 1734-1742.

41. Machungo C, Losenge T, Kahangi E, Coyne D, Dubois T, et al. (2009) Effect of endophytic Fusarium oxysporum on growth of tissuecultured banana plants. African Journal of Horticultural Science 2.

42. Nassar AH, EL-Tarabily KA, Sivasithamparam K (2005) Promotion of plant growth by an auxin-producing isolate of the yeast Williopsis saturnus endophytic in maize (Zea mays L.) roots. Biology and Fertility of soils 42 : 97-108.

43. Hussain A, Shah ST, Rahman H, Irshad M, Iqbal A (2015) Effect of IAA on in vitro growth and colonization of Nostoc in plant roots. Frontiers in plant science 6: 46. 